\title{
Eulerian Formulation for an Extensible Elastic Rod
}

\author{
A. Huynen \\ F.R.I.A., F.R.S.-FNRS, National Fund for Scientific Research, Bruxelles, Belgium \\ Department of Civil Engineering, University of Minnesota, USA
}

E. Detournay

Department of Civil Engineering, University of Minnesota, USA

\author{
V. Denoël \\ Structural Engineering, Department of Architecture, Geology, Environment and Constructions \\ University of Liege, Belgium
}

\begin{abstract}
This paper reformulates the governing equations of an extensible elastic rod by reference to a given spatial curve. This Eulerian formulation is motivated by the need to solve efficiently the constrained elastica problem encountered in many medical and engineering applications, in which a thin rod is inserted in a tortuous conduit. The Eulerian reformulation of the equations hinges on the restatement of the rod local equilibrium in terms of derivatives with respect to the curvilinear coordinate associated with the reference curve and the description of the rod deflection as a perturbation of this curve. The originality of the proposed formulation lays in the axially unconstrained character of the resulting system such that the determination of the rod configuration between two fixed points reduces to the resolution of a classical boundary value problem.
\end{abstract}

\section{INTRODUCTION}

The general problem of a slender elastic body constrained to deform inside a straight or sinuous conduit is widely encountered in medical and engineering applications. Examples include the insertion of miniature instruments into blood vessels for the treatment of vascular and cerebrovascular pathologies (e.g. abdominal and thoracic aortic aneurism, stroke) as well as the endoscopic examination of internal organs (e.g. colonoscopy, fibroscopy, rhinoscopy), see for instance Schneider (2003). Another application concerns the petroleum industry which relies on several kilometers long drillstrings to transmit the axial force and torque necessary to drill the rock formations and reach deep hydrocarbon reservoirs (Inglis 1988, Sampaio 2008). The assessment of the energy loss along the drill string, known as the torque and drag problem, plays an essential role in drilling and well design as the friction appearing at the contacts between the drillstring and the borehole may dramatically increase the costs or, even, be a limiting factor in some configurations (Mason and Chen 2007). The identification of the number of contacts between the rod and the conduit (e.g. blood vessel or borehole) as well as their position and extent constitutes the central concern of this relatively broad class of problems. The nonlin- earities associated with the large deflection of the rod and the unilateral contact condition as well as the $a$ priori unknown number of contacts, however, make the use of conventional numerical tools rather inefficient. Additionally, the commonly adopted division of the problem in rod segments bounded by two contacts (Chen \& Li 2007, Denoël 2008) requires to solve the governing equations on domains which are initially unknown and, therefore, leads to the establishment of integral constraints on the unknown length of a rod forced to go through fixed points in space. Finally, the assessment of the unilateral contact condition, which requires in principle the comparison of two curves parametrized by distinct curvilinear coordinates (e.g. the rod centroid and the conduit axis), prove to be a rather intensive computational task.

To circumvent the above-mentioned difficulties, an Eulerian reformulation of the rod governing equations is proposed and the strategy initiated by Denoël \& Detournay (2011) for the planar elastica is generalized to three-dimensional configurations and extensible rods. Modeling the elastic body by means of the special Cosserat rod theory, its deflection is described as a perturbation of a reference curve (e.g. the conduit axis) while the rod local equilibrium is formulated within the Eulerian reference frame associated with this curve. Consequently, the determination of the rod 
configuration between two adjacent contacts reduces to the resolution of a classical boundary value problem for which, in addition to the axial components of the internal force and moment at one end, the rod eccentricity as well as its inclination with respect to the reference curve are prescribed at both extremities of the domain.

The originality of the proposed formulation lays in the axially unconstrained character of the resulting system. Indeed, as the reference curve is substituted for the domain of the problem, the unstressed length of the rod is not prescribed and, therefore, freely evolves as a result of the loading. This particular aspect is encountered in various additional applications in which the length of the domain is a priori unknown or susceptible to self-adjustment such as the continuous casting process, the extrusion of plastic through a draw plate or the laying of cables and pipelines. We will refer to the ability of the proposed method to handle this particular class of axially unconstrained problems, as self-feeding.

\section{PROBLEM DEFINITION \& GOVERNING EQUATIONS}

The canonical problem considered in this paper consists in the determination of the deformed configuration adopted by an extensible rod forced to go through two fixed points in space and subjected to a known external loading (e.g. weight, applied torque and tension at an extremity). Consequently, the length of the rod, which satisfies the boundary conditions and is in equilibrium with the external loading, is unknown.

To solve this problem, let us define the right-handed orthonormal basis $\left\{\boldsymbol{e}_{k}\right\}$ for the Euclidean space $\mathbb{E}^{3}$ and denote by $\boldsymbol{R}(S)=X_{k} \boldsymbol{e}_{k}$ the position vector of the point lying on the reference curve $\mathscr{C}$ at $S$. The arclength parameter $S$, referred to as the Eulerian coordinate, identifies a section along $\mathscr{C}$ which consists of all points whose reference positions are on the plane perpendicular to the reference curve at $S$. For the sake of clarity, the parametrization of this curve is assumed to be natural such that $\mathscr{C}$ is said to be unit-speed (Pressley 2010) .

Having defined the global $\left\{\boldsymbol{e}_{k}\right\}$-basis, the position vector of the rod cross section centroid with curvilinear coordinate $s$ is denoted by $\boldsymbol{r}(s)=x_{k} \boldsymbol{e}_{k}$. The parameter $s$, which is referred to as the (natural) Lagrangian coordinate in contrast to the Eulerian coordinate $S$, identifies the rod cross section in its unstressed configuration $r^{0}(s)$. To fully characterize the spatial configuration of the rod, one has to additionally supply this space curve $\mathscr{E}$ with a pair of orthonormal vectors $\boldsymbol{d}_{1}(s), \boldsymbol{d}_{2}(s)$ characterizing the configuration of a pair of orthogonal material lines of the section (Antman 2005). The rod is therefore entirely defined by the three-vector valued functions

$\left[s_{a}, s_{b}\right] \ni s \mapsto \boldsymbol{r}(s), \boldsymbol{d}_{1}(s), \boldsymbol{d}_{2}(s) \in \mathbb{E}^{3}$ with $s=s_{a}$ corresponding to the origin of the canonical problem located at $S=S_{a}$, and $s=s_{b}$ corresponding to its extremity located at $S=S_{b}$, see Figure 11(a).

The cross section orientation is then naturally described by its normal $\boldsymbol{d}_{3}(s)=\boldsymbol{d}_{1} \times \boldsymbol{d}_{2}$ such that the resulting triplet of directors $\left\{\boldsymbol{d}_{k}(s)\right\}$ constitutes a right-handed orthonormal basis for $\mathbb{E}^{3}$ for each cross section $s$. The rod change of shape is measured by the twist and stretch vectors, $\boldsymbol{u}=u_{k} \boldsymbol{d}_{k}$ and $\boldsymbol{v}=v_{k} \boldsymbol{d}_{k}$, which are defined as
$\mathrm{d}_{s} \boldsymbol{d}_{k}=\boldsymbol{u} \times \boldsymbol{d}_{k}$
$\mathrm{d}_{s} \boldsymbol{r}=\boldsymbol{v}$

respectively. Their components in the directors basis, $u_{k}$ and $v_{k}$, are referred to as strain variables.

Considering only local interactions between adjacent cross sections of the rod and defining the internal force $\boldsymbol{F}(s)=F_{k} \boldsymbol{d}_{k}$ and moment $\boldsymbol{M}(s)=M_{k} \boldsymbol{d}_{k}$, the conservation of linear and angular momenta yields (Antman 2005)

$$
\begin{array}{r}
\mathrm{d}_{s} \boldsymbol{F}+\boldsymbol{f}=0 \\
\mathrm{~d}_{s} \boldsymbol{M}+\mathrm{d}_{s} \boldsymbol{r} \times \boldsymbol{F}=0
\end{array}
$$

where $\boldsymbol{f}(s)=w \boldsymbol{e}_{3}$ is the body force per unit reference length with $w$ the rod specific weight.

For an unshearable rod of circular cross section made of linearly elastic material, the components of the internal force and moment are related to the strain variables by means of the following constitutive equations

$$
\begin{gathered}
\boldsymbol{M}(s)=E I\left(u_{1} \boldsymbol{d}_{1}+u_{2} \boldsymbol{d}_{2}\right)+G J u_{3} \boldsymbol{d}_{3} \\
\boldsymbol{F}(s)=F_{1} \boldsymbol{d}_{1}+F_{2} \boldsymbol{d}_{2}+E A(\alpha-1) \boldsymbol{d}_{3}
\end{gathered}
$$

with the bending stiffness $E I$, the torsional stiffness $G J$ and the axial stiffness $E A$. To close the formulation, one must additionally consider the material restraint $\boldsymbol{v}(s)=\alpha \boldsymbol{d}_{3}$ which expresses that the centroid tangent vector is aligned with the normal to the rod cross section and where the stretch $\alpha(s)>0$.

\subsection{Parametrization of the rotation group $S O(3)$}

The use of six components for the directors $\boldsymbol{d}_{1}$ and $\boldsymbol{d}_{2}$ may appear clumsy since these components are subject to three orthonormality constraints. The introduction of unit quaternions to parametrize the threedimensional rotation group $S O(3)$ is an alternative to the habitual Euler angles subject to gimbal lock (Kuipers 1999). It is motivated by the desire to resort to a regular representation of the rotation tensor.

According to Euler's rotation theorem, the orientation of the triplet of directors $\left\{\boldsymbol{d}_{k}(s)\right\}$ can be defined by specifying its rotation about the unit axis of rotation $\boldsymbol{a}$ through the angle $\omega$ with respect to the global $\left\{\boldsymbol{e}_{k}\right\}$-basis. The set $\{\omega, \boldsymbol{a}\}$ can advantageously be replaced by the so-called quaternion 

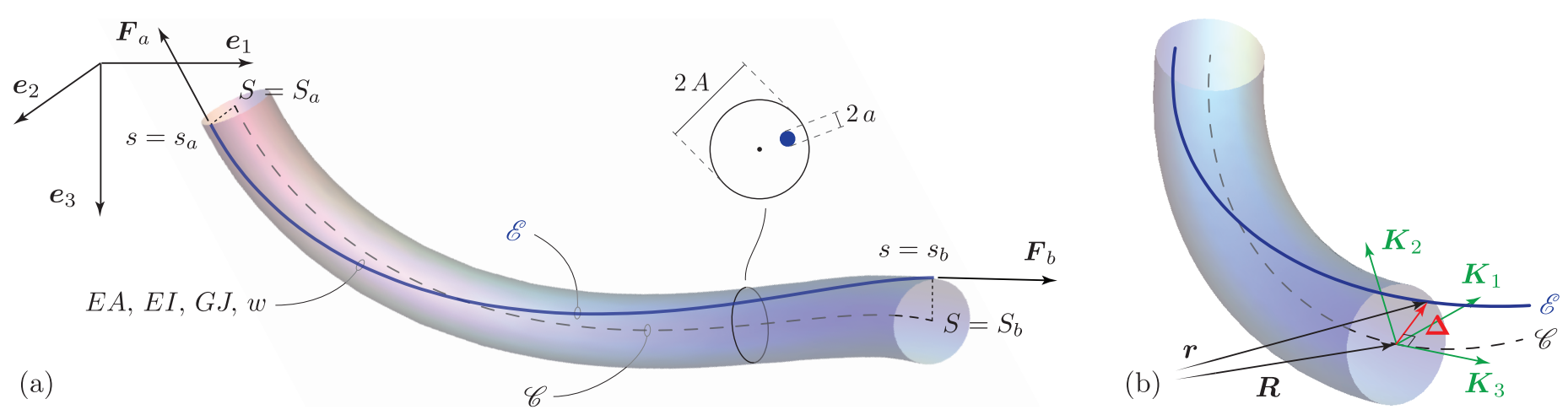

Figure 1: (a) Description of the canonical problem, and (b) Decomposition of the position vector $\boldsymbol{r}(s)=\boldsymbol{R}(S)+\boldsymbol{\Delta}(S)$. For the sake of readability, the region in which the solution is expected be found is represented (for the constrained problem, this region corresponds to the conduit centered on the reference curve).

$\mathfrak{g}(s)=\left(g_{0}, g_{1}, g_{2}, g_{3}\right)^{T}$ with $\|\mathfrak{g}\|=1$ and whose components are defined as

$g_{0}(s)=\cos (\omega / 2) \quad \boldsymbol{g}(s)=\sin (\omega / 2) \boldsymbol{a}$

(Kuipers 1999). Additionally to being regular, the quaternion components vary continuously as the orientation changes.

\subsection{Shortcomings of the Lagrangian formulation}

As already emphasized in the introduction, the canonical problem under consideration consists in a two points boundary value problem for which the rod location and inclination are prescribed at both extremities $s_{a}$ and $s_{b}$ of the domain. However, as the parametric coordinates of the rod $x_{k}(s)$ in the absolute reference frame $\left\{\boldsymbol{e}_{k}\right\}$ are given by

$x_{k}(s)=x_{k}\left(s_{a}\right)+\int_{s_{a}}^{s} \mathrm{~d}_{s} \boldsymbol{r} \cdot \boldsymbol{e}_{k} \mathrm{~d} s$

with $\mathrm{d}_{s} \boldsymbol{r}=\boldsymbol{v}$, the boundary conditions associated with the canonical problem reduce to a set of three integral constraints on the unknown length of the rod forced to go through two fixed points in space. These stiff isoperimetric constraints result in ill-conditioned equations requiring an excessive amount of iterations, provided that convergence is reached, which worsens with decreasing bending stiffness (Denoël \& Detournay 2011).

For the constrained problem, a further difficulty is related to the assessment of the unilateral contact condition which ensures that the computed deformed configuration of the rod is contained within the conduit. This constraint indeed requires the evaluation of the distance $\Delta$ between the reference curve (i.e. the conduit axis), parametrized as

$X_{k}(S)=X_{k}\left(S_{a}\right)+\int_{S_{a}}^{S} \mathrm{~d}_{S} \boldsymbol{R} \cdot \boldsymbol{e}_{k} \mathrm{~d} S$

in the absolute reference frame $\left\{\boldsymbol{e}_{k}\right\}$, and the rod cross section centroid $\mathscr{E}$ given in Equation (8). As these two curves are parametrized by two distinct curvilinear coordinates ( $S$ and $s$ respectively), this task turns out to be computationally intensive.

Finally, the ill-conditioning of the governing equations for small flexural rigidity, the existence of spurious solutions associated with curling of the rod as well as the demand for increasing accuracy of the solutions with decreasing clearance, are sources of various additional complications or other bottlenecks, with the consequence that the Lagrangian formulation of the problem is particularly ineffective and laborious to solve.

\section{EULERIAN FORMULATION}

To circumvent the above-mentioned shortcomings of the Lagrangian formulation and on account of the nature of the problem, which is characterized by relatively close spatial curves $\mathscr{C}$ and $\mathscr{E}$, the proposed formulation hinges on the introduction of the eccentricity vector $\boldsymbol{\Delta}(S)$, characterizing the rod deflection with respect to the reference curve. The description of the problem is further expressed in terms of the Eulerian coordinate $S$ rather than the natural Lagrangian coordinate $s$ of the rod. As presented in Figure 1(b), the position vector of the rod cross section centroids $\boldsymbol{r}(s)=x_{k} \boldsymbol{e}_{k}$ can, naturally, be expressed as

$\boldsymbol{r}(s)=\boldsymbol{R}(S)+\boldsymbol{\Delta}(S)$

where the position vector of the reference curve $\boldsymbol{R}(S)=X_{k} \boldsymbol{e}_{k}$ is known, and the eccentricity vector is perpendicular to $\mathscr{C}$, i.e. $\mathrm{d}_{S} \boldsymbol{R} \cdot \boldsymbol{\Delta}=0$. The former expression describes the rod configuration as a perturbation of the reference curve, such that the eccentricity vector can be interpreted as a measure of the rod deflection and rotation with respect to $\mathscr{C}$ in the section of curvilinear coordinate $S$.

From now on, derivatives of scalar and vector valued functions with respect to the Eulerian coordinate $S$ will be denoted by the apposition of a prime while derivatives with respect to the Lagrangian coordinate $s$ are explicitly specified. 


\subsection{Definition of the reference curve}

With each point of the reference curve, one may associate a triplet $\left\{\boldsymbol{K}_{j}(S)\right\}$ constituting a right-handed orthonormal basis with $\boldsymbol{K}_{3}=\boldsymbol{R}^{\prime}$ defined as the unit vector tangent to $\mathscr{C}$. The orientation of this triplet can be defined through a unit quaternion $\mathfrak{Q}(S)$ specifying its rotation with respect to the global $\left\{\boldsymbol{e}_{j}\right\}$-basis. Additionally imposing the component of this rotation about $\boldsymbol{K}_{3}$ to be null, one has

$\mathfrak{Q}(S)=\left(Q_{0}, Q_{1}, Q_{2}, 0\right)^{T}$

with $Q_{0}^{2}+Q_{1}^{2}+Q_{2}^{2}=1$. This arbitrary definition of $Q_{3}(S)=0$ simply reflects that the reference curve needs only to be specified through the inclination of its tangent vector $\boldsymbol{K}_{3}(S)$ on the global axes; in other words the possible rotation of a pair of vectors spanning the plane perpendicular to the reference curve is irrelevant. An alternative definition of the unit quaternion $\mathfrak{Q}(S)$ would be to specify its components such that the triplet $\left\{\boldsymbol{K}_{j}(S)\right\}$ coincides with the Frenet basis attached to the reference curve. However this would lead to uniqueness and continuity issues for reference curves with vanishing curvature.

As a result of the previous definition, the pair $\left\{\boldsymbol{K}_{1}, \boldsymbol{K}_{2}\right\}$ constitutes an orthonormal basis for the plane perpendicular to the reference curve in which the eccentricity vector reads

$$
\boldsymbol{\Delta}(S)=\Delta_{1} \boldsymbol{K}_{1}+\Delta_{2} \boldsymbol{K}_{2}
$$

with $\Delta_{1}=\Delta \cos \beta$ and $\Delta_{2}=\Delta \sin \beta$. The components of the position vector in the global basis may, therefore, be expressed as

$$
\begin{aligned}
& x_{1}(s)=X_{1}+\Delta_{1}\left(1-2 Q_{2}^{2}\right)+2 \Delta_{2} Q_{1} Q_{2} \\
& x_{2}(s)=X_{2}+2 \Delta_{1} Q_{1} Q_{2}+\Delta_{2}\left(1-2 Q_{1}^{2}\right) \\
& x_{3}(s)=X_{3}-2 Q_{0}\left(\Delta_{1} Q_{2}-\Delta_{2} Q_{1}\right)
\end{aligned}
$$

in which each variable is a function of the Eulerian coordinate $S$ and where the $X_{j}$ 's are obtained by integration of the reference curve local inclination expressed in terms of the quaternion components $Q_{j}(S)$.

\subsection{Definition of the rod}

By analogy with the definition of the reference curve and for reasons that will become clear in the following section, the orientation of the rod cross section is described by means of two unit quaternions

$$
\mathfrak{q}(s)=\left(q_{0}, q_{1}, q_{2}, 0\right)^{T} \quad \mathfrak{t}(s)=\left(t_{0}, 0,0, t_{3}\right)^{T}
$$

representing two successive rotations and such that the quaternion corresponding to the total rotation is given by the quaternion product $\mathfrak{g}(s)=\mathfrak{q} \cdot \mathfrak{t}$. This parametrization of the rotation tensor allows to uncouple the attitude of the director $\boldsymbol{d}_{3}(s)$ from the rotation of the section about this director.

Expressing the directors in terms of the unit quaternion $\mathfrak{g}(s)$, one may compute their derivatives $\mathrm{d} \boldsymbol{d}_{k} / \mathrm{d} s$ and identify the components of the twist vector, $\boldsymbol{u}=$ $u_{k} \boldsymbol{d}_{k}$, in the directors basis.

\subsection{Jacobian of the mapping}

To complete the reformulation of the local equilibrium (3, 4) within the reference frame associated to $\mathscr{C}$, one has to further express the natural derivatives $\mathrm{d} \cdot / \mathrm{d} s$ in terms of derivatives with respect to the Eulerian curvilinear coordinate $\mathrm{d} \cdot / \mathrm{d} S$. The function $s(S)$, which maps the Eulerian coordinate of the reference curve on the Lagrangian coordinate of the rod, is therefore introduced. Considering the definition (2) of the centroid tangent vector $\mathrm{d} \boldsymbol{r} / \mathrm{d} s$ in terms of the stretch vector components and applying the chain rule differentiation, the Jacobian $s^{\prime}(S)$ of this transformation can be expressed as

$$
\frac{\mathrm{d} s}{\mathrm{~d} S}= \pm\left\|\boldsymbol{R}^{\prime}+\boldsymbol{\Delta}^{\prime}\right\| /\|\boldsymbol{v}\|
$$

where the positive sign is selected in order to prevent the appearance of parasitic solutions associated with curling of the rod. This expression emphasizes that the origin of the drift existing between the two curvilinear coordinates is two-fold: ( $i$ ) the eccentricity between the rod and the reference curve, and (ii) the stretch $\|\boldsymbol{v}\|=\alpha$ of the rod itself.

Defining the function $J_{1}(S)=1 / s^{\prime}$ as the Jacobian of the inverse mapping $S(s)$, i.e. from the Lagrangian to the Eulerian coordinate, one may compute its successive derivatives using the following recursive relation

$J_{k}(S)=\left.\frac{\mathrm{d}^{k} S}{\mathrm{~d} s^{k}}\right|_{s=s(S)}=J_{k-1}^{\prime} J_{1}$

for $k>1$.

\subsection{Rod attitude}

The next step in the Eulerian reformulation of the problem consists in rewriting the quaternion $\mathfrak{g}(s)$, representing the directors orientation, in terms of the quaternion $\mathfrak{Q}(S)$, expressing the reference curve local inclination, and the components $\left(\Delta_{1}, \Delta_{2}\right)$ of the eccentricity vector in the section $S$.

Using expressions $(13-15)$ for the position vector components and the definitions of the directors $\left\{\boldsymbol{d}_{k}\right\}$ in terms of the quaternion $\mathfrak{g}=\mathfrak{q} \cdot \mathfrak{t}$ in the kinematic relation (2), one may obtain the following set of equa- 
tions

$$
\begin{array}{r}
2 \alpha q_{0} q_{2}=J_{1} \boldsymbol{r}^{\prime} \cdot \boldsymbol{e}_{1} \\
-2 \alpha q_{0} q_{1}=J_{1} \boldsymbol{r}^{\prime} \cdot \boldsymbol{e}_{2} \\
\alpha\left(2 q_{0}^{2}-1\right)=J_{1} \boldsymbol{r}^{\prime} \cdot \boldsymbol{e}_{3}
\end{array}
$$

with the stretch $\alpha>0$ and where the uncoupling between the representation of $\boldsymbol{d}_{3}$ attitude and the rotation of the section about this director has been advantageously used. Solving this set of equations, one may relate the Lagrangian quantities $q_{j}(s)$ function of the rod curvilinear coordinate $s$ to the Eulerian quantities $Q_{j}(S)$ and $\Delta_{1}(S), \Delta_{2}(S)$ function of the reference curve curvilinear coordinate $S$.

\subsection{The excess of twist}

Finally, the second quaternion $\mathfrak{t}(s)$ characterizing the rotation of the rod cross section about the director $\boldsymbol{d}_{3}(s)$ cannot be related to the eccentricity vector and, therefore, to the Eulerian quantities $\Delta_{1}(S)$ and $\Delta_{2}(S)$. The angle $\varphi(S)$ is consequently introduced such that $t_{0}=\cos (\varphi / 2)$ and $t_{1}=\sin (\varphi / 2)$ with

$$
\varphi_{, s}=J_{1} \varphi^{\prime} \quad \varphi_{, s s}=J_{1}^{2} \varphi^{\prime \prime}+J_{2} \varphi^{\prime}
$$

and where $\varphi_{, s}$ can be interpreted as the internal torsion or the excess of twist of the rod. Similarly, additional Lagrangian quantities appearing in the conservation of linear and angular momenta, such as the internal force $\boldsymbol{F}(s)$ or the stretch $\alpha(s)$, are re-written as functions of the Eulerian coordinate and their derivatives are evaluated using the same scheme.

\subsection{Boundary conditions}

In conclusion, one obtains a mixed order system of six ODE's involving exclusively Eulerian quantities. This system being first order in the components $F_{k}(S)$ of the internal force, second order in the angle $\varphi(S)$, and third order in the components $\Delta_{k}(S)$ of the eccentricity vector, a total of eleven boundary conditions is required.

In addition to the rod eccentricity and inclination with respect to the reference curve, which must be prescribed at both extremities of the boundary value problem, the axial component of the internal force and the torque need to be specified at one extremity of the domain. The eccentricity vector $\boldsymbol{\Delta}(S)$ and its derivative $\boldsymbol{\Delta}^{\prime}(S)$ are, therefore, imposed at both $S=S_{a}$ and $S=S_{b}$ while the stretch $\alpha(S)$ as well as the angle $\varphi(S)$ and its derivative $\varphi^{\prime}(S)$ are prescribed at either $S=S_{a}$ or $S=S_{b}$, depending on the nature of the problem under consideration.

\section{NUMERICAL IMPLEMENTATION}

To solve numerically the resulting nonlinear boundary value problem, a collocation method has been implemented. Defining a partition $\pi$ of $\left[S_{a}, S_{b}\right]$ constituted of $N$ subintervals, we seek an approximate solution $\left(\boldsymbol{\Delta}^{*}, \varphi^{*}, \boldsymbol{F}^{*}\right)$ such that

$$
\begin{aligned}
\Delta^{*} & \in \mathcal{P}_{k+3, \pi} \cap C^{2}\left[S_{a}, S_{b}\right] \\
\varphi^{*} & \in \mathcal{P}_{k+2, \pi} \cap C^{1}\left[S_{a}, S_{b}\right] \\
\boldsymbol{F}^{*} & \in \mathcal{P}_{k+1, \pi} \cap C^{0}\left[S_{a}, S_{b}\right]
\end{aligned}
$$

where $k \geq 3$ is the number of collocation points per subinterval and $\mathcal{P}_{n, \pi}$ is the set of all piecewise polynomial functions of order $n$ on the partition $\pi$. For reasons of efficiency, stability, and flexibility in order and continuity, $B$-splines are chosen as basis functions while collocation is applied at Gaussian points (Ascher et al. 1979).

The unknown $B$-splines coefficients are then determined by solving the nonlinear system of $6 k N$ collocation equations and imposing the 11 boundary conditions. This method has been implemented in MATLAB using the nonlinear solver fsolve as well as the Spline Toolbox (De Boor 2005) to evaluate the $B$ splines and their derivatives.

\section{APPLICATION}

As an implementation of the proposed formulation, we consider the progressive twisting of a clampedclamped beam subject to its specific weight $w$ as well as a prescribed tension $T$, see Figure 2(a). The particularity of the present loading, which results from the Eulerian reformulation of the problem, is that the unstressed length of the rod $\ell^{0}$ is actually not prescribed but freely evolves as the loading progress (c.f. selffeeding); the length $L$ of the straight reference curve remaining however constant.

The response diagram presented in Figure 2 pictures the twist angle $\varphi(L)$, imposed at the rod extremity, versus the dimensionless stretched length $(\ell-L) / L$ for various values of the loading parameter $\Gamma=T / w L$. This diagram was obtained for a unitary distance between supports, i.e. $L=1$, and a specific weight $w=10$ while the rod stiffnesses were chosen such that $E I=4 G J / 3=E A / 255=1$. These relations as well as the deformed configurations graphed in the figure were obtained using the numerical implementation presented in the previous section with $k=5$ gaussian points in each of the $N=15$ uniformly spaced subintervals.

Observe that, while the number of helical waves in the deformed configuration clearly increases with the imposed end rotation $\varphi(L)$, foldings in the relation between the end rotation and the dimensionless stretched length are triggered by the development of 


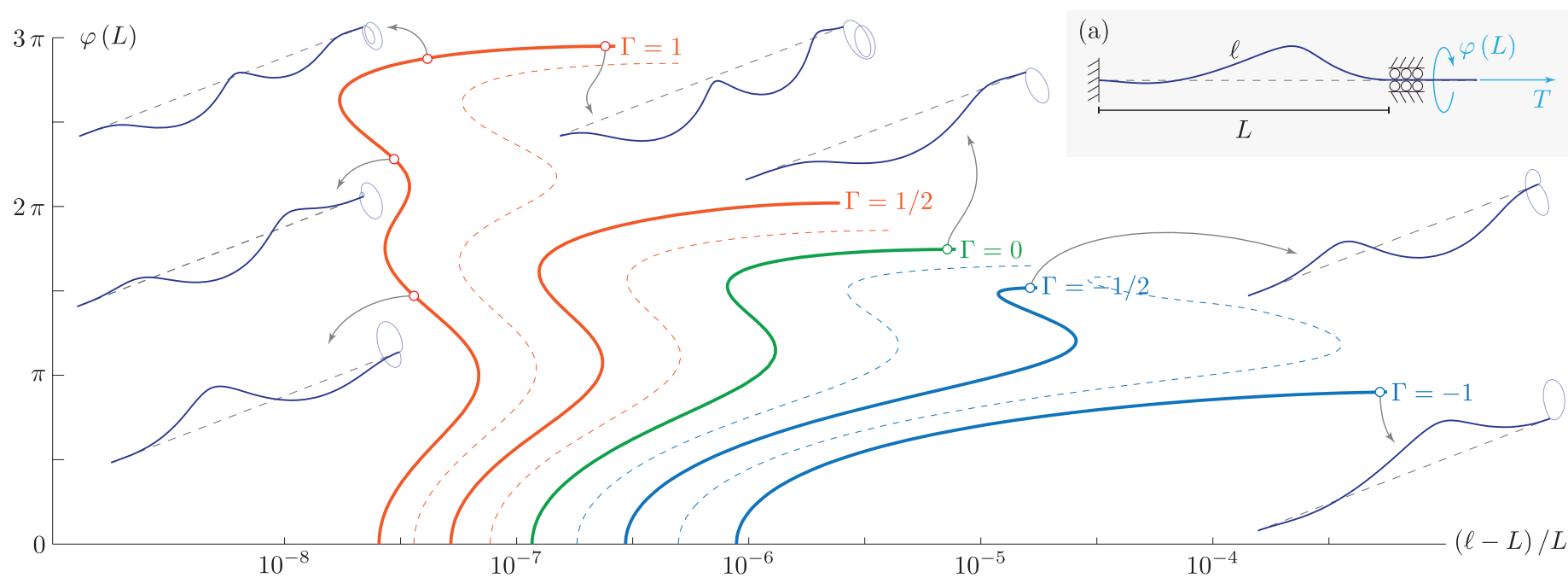

Figure 2: Response diagram of a clamped-clamped beam subject to progressive twisting as well as its specific weight $w=10$ and a prescribed tension $T$. The imposed end rotation $\varphi(L)$ is plotted as a function of the dimensionless stretched length $(\ell-L) / L$ for various values of the loading parameter $\Gamma=T / w L$ and a unitary distance between support $L=1$; the rod stiffnesses were chosen such that $E I=4 G J / 3=E A / 255=1$.

additional helical waves in the rod deformed configuration. Eventually, material flows inside the domain either for compressive axial force (buckling) or insufficient tensile force (regarding the imposed end rotation) as more rod is required to maintain the equilibrium state.

\section{CONCLUSIONS}

The Eulerian reformulation of the equations governing the three-dimensional deflection of an extensible rod has been achieved by $(i)$ the introduction of the eccentricity vector $\Delta(S)$, characterizing the rod deflection with respect to a given reference curve, (ii) the uncoupling of the attitude of the rod centroid tangent vector form the rotation of the cross section about it, and (iii) the expression of the rod local equilibrium in terms of derivatives with respect to the reference curve curvilinear coordinate. The originality of the proposed formulation, which resolves in one stroke a series of issues that afflict the classical Lagrangian approach (isoperimetric constraints and potential contact detection with the wall of a conduit), lays in the self-feeding character of the resulting system as the unstressed length of the rod is subject to self-adjustement in response to the loading. Finally, the response of a clamped-clamped beam subject to progressive twisting is obtained using the proposed formulation and its numerical implementation.

\section{Acknowledgement}

The authors acknowledge the National Fund for Scientific Research of Belgium for its support.
Ascher, U., J. Christiansen, \& R. D. Russell (1979). A collocation solver for mixed order systems of boundary value problems. Mathematics of Computation 33(146), 659-679.

Chen, J.-S. \& C.-W. Li (2007). Planar elastica inside a curved tube with clearance. International Journal of Solids and Structures 44(18-19), 6173-6186.

De Boor, C. (2005). Spline toolbox for use with MATLAB: user's guide, version 3. MathWorks.

Denoël, V. (2008). Advantages of a semi-analytical approach for the analysis of an evolving structure with contacts. Communications in Numerical Methods in Engineering 24(12), 1667-1683.

Denoël, V. \& E. Detournay (2011). Eulerian formulation of constrained elastica. International Journal of Solids and Structures 48(3-4), 625 - 636.

Inglis, T. (1988). Directional drilling, Volume 2. Springer.

Kuipers, J. (1999). Quaternions and Rotation Sequences: A Primer With Applications to Orbits, Aerospace, and Virtual Reality. Princeton Univers. Press.

Mason, C. \& D. Chen (2007). Step changes needed to modernise T\&D software. In SPE/IADC Drilling Conference.

Pressley, A. (2010). Elementary Differential Geometry. Springer London.

Sampaio, J. H. B. (2008). Drilling Engineering. Curtin University of Technology, Department of Petroleum Engineering.

Schneider, P. (2003). Endovascular skills: guidewire and catheter skills for endovascular surgery. CRC Press.

\section{REFERENCES}

Antman, S. (2005). Nonlinear problems of elasticity, Volume 107. Springer Verlag. 